\title{
Diagnosing Early or Rheumatoid Arthritis. Which Is Better: Expert Opinion or Evidence?
}

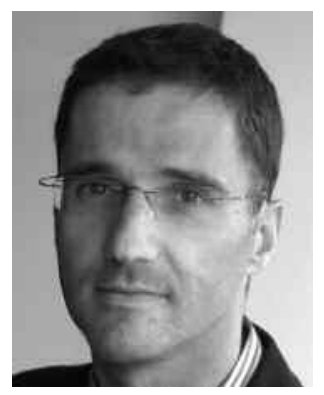

All clinicians know about the complexity in diagnosing rheumatoid arthritis (RA), especially early in the disease. Because RA lacks pathognomonic features - that is, there are no clinical, biological, or radiological characteristics specific to RA diagnosis - doubt about the diagnosis may persist for some patients ${ }^{1,2}$. Examples are patients with "nude" polyarthritis [i.e., without positivity for serum rheumatoid factor (RF), anti-citrullinated peptide antibodies, typical erosion, or all 3], or even elderly people with erosive RF-positive polyarthritis associated with psoriasis or calcium crystal deposition disease features seen on joint radiography. When RA is neither obvious nor completely excluded, the clinician strikes a balance between possible or probable RA, depending on the level of confidence. In this context, in clinical research, RA classification criteria may be of some help because they ensure, at the group level, the diagnosis of RA with minimal error. However, in clinical practice, RA criteria cannot be used as the gold standard, especially in early arthritis (EA), as was previously shown $n^{3-5}$.

In this issue of The Journal, Morvan, et al report on a cohort of patients with EA followed for 10 years to investigate discrepancies in RA diagnosed by American College of Rheumatology (ACR) classification criteria and final diagnosis by an office-based rheumatologist ${ }^{6}$. The authors noted poor agreement at the onset of the disease, as has been shown, but also at 2 years, when ACR criteria are supposed to be more accurate. If one assumes that the rheumatologist is an expert, who is right: the expert or the criteria? Eminence versus evidence?

Morvan and colleagues assume that to improve sensitivity and specificity, the ACR criteria need to be "adjusted" with rheumatologist expertise evidence, that is, the diagnosis made by an office-based rheumatologist. The development of classification criteria involves experts who express their opinion about the final diagnosis for a sample of patients with the targeted disease, RA in this case, or other diseases supposed to be potential differential diagnoses. In the development of the 1987 ACR classification criteria, controls had different sorts of rheumatic diseases, especially osteoarthritis, whose expression differs greatly from that of RA. This limitation was acknowledged in the development of the ACR criteria ${ }^{7}$. Moreover, even if a substantial number of patients were included in the development of the ACR criteria, their diseases encompass only a subset of rheumatologic diseases and not the whole spectrum of RA, or its differential diagnoses. Thus, the list of items resulting from the development of the ACR classification criteria likely reflected only the most usual features of RA and probably not atypical presentations ${ }^{4,8}$. At the patient level, the clinician likely considers other relevant characteristics favoring or not favoring RA diagnosis, the main reason for which Medicine is often compared to Art.

The question, then, is how to incorporate clinical experience in classification criteria, in other words, to join expertand evidence-based medicine. Because in daily clinical practice physicians make a diagnosis on the basis of signs or symptoms in favor of a given diagnosis on the one hand, and the absence of features in favor of other differential diagnoses on the other, the definition of exclusion criteria could help close the gap between criteria and expert opinion. When used, exclusion criteria may represent uncommon clinical situations, probably not found in the control group, used to develop the set of criteria but recognized by experts to be misleading. Such is the case with classification criteria for Sjögren's syndrome or adult Still's disease, for example ${ }^{9,10}$. Still, exclusion criteria are necessarily limited in number and thus remain somehow restricted as compared with clinician experience.

Another option could be to associate - rather than integrate - the classification criteria with a level of confidence or certainty with regard to the targeted diagnosis, for example, RA. The development of the 1987 ACR classification criteria involved such an evaluation ${ }^{8}$ : a $10-\mathrm{cm}$ visual analog scale, where 0 represented the absolute certainty that the

See Changes over time in the diagnosis of RA, page 2428. 
patient did not have RA and 100 the absolute certitude that the patient did. The resulting certainty score was 89 , on average, for RA patients and 6 for controls. The report by Morvan and colleagues did not point out, at least for some RA patients or controls, strong disagreement between classification criteria and the certainty score.

On the basis of this experience, as well as knowledge of the poor performance of RA classification criteria in EA, we recorded such a score at each visit for patients included in the ESPOIR cohort, the EA cohort developed in France since $2002^{11}$. At inclusion, $71 \%$ of included patients satisfied the 1987 ACR criteria ${ }^{11}$. When using the certainty VAS with a score $>75$, we could identify 49 additional patients at their inclusion in the cohort ${ }^{12}$; all these patients subsequently received a diagnosis of RA, with, as a gold standard, satisfaction of the 1987 ACR criteria at the 2-year visit. However, certainty scales are considered too subjective for many authors or reviewers, and their use in studies remains limited.

In the study by Morvan and associates, clinicians were asked to note their diagnosis for each patient, but they did not have to provide the level of confidence in this diagnosis or the possibility of an alternative diagnosis. If one assumes that the diagnosis reported by the rheumatologist is the result of clinical expertise, then neither the expertise, nor the classification criteria is accurate enough to diagnose RA early in the disease. At baseline, ACR criteria lack sensitivity, as shown ${ }^{3}$. Expert-based diagnosis also lacks specificity. This situation highlights the current trend favoring the terms "persistent arthritis" and/or "erosive arthritis" for EA instead of RA and subsequently to make treatment decisions on this basis, that is, to initiate disease-modifying antirheumatic drugs (DMARD) and not on the more specific diagnosis that will need more time to assess ${ }^{2,13,14}$. Two years after disease onset, both ACR criteria and clinician expertise seem to perform better in diagnosis, especially when combined. Therefore, 2 years might be reasonable for an accurate diagnosis and to move from a diagnosis of persistent and/or erosive arthritis to RA. The above interpretation is of course debatable, because Morvan and colleagues did not report an external validation of the final diagnosis. Thus, one could argue that the apparent stability of the office-based rheumatologist diagnosis is more a reflection of the reluctance of clinicians to change their minds once a diagnosis is made and explained to the patient, and a specific therapeutic strategy adopted. Another issue is the impact of RA treatment on the natural history of the disease. As shown in the PROMPT study, methotrexate started early in patients with EA is likely to prevent the onset of typical and complete $\mathrm{RA}^{15}$. This situation is probably similar with respect to other efficient DMARD or biologics and might have explained the performance of the ACR criteria described by Morvan and colleagues. Alternatively, such patients may have been more likely to stop visiting the rheumatologist and to have been among the numerous people lost to followup at the 10-year assessment.

The debate on expert- versus evidence-based diagnosis is not closed. The lack of an easy option reinforces the consensus recommending treatment decisions based not directly on the diagnosis but, rather, on the risks that may evolve throughout the course of the disease. With EA, the key objective is to determine whether the disease will be persistent or self-limiting, which will allow rapid initiation of a DMARD able to control inflammation and block structural damage, whatever the exact nature of the rheumatism. At a more established stage, more accuracy is required to determine the longterm risks associated with the disease and, subsequently, the optimal therapeutic strategy.

\section{BRUNO FAUTREL, MD, PhD,}

APHP, Groupe hospitalier Pitié-Salpêtrière, Service de Rhumatologie,

Université Paris 6, Pierre et Marie Curie, UFR de médecine,

Paris, F-75013 France

Address correspondence to Dr. B. Fautrel, Department of Rheumatology, Pitié-Salpêtrière Hospital, 83 boulevard de l'Hôpital, 75651 Paris cedex 13, France.E-mail: bruno.fautrel@psl.aphp.fr

Acknowledgement The author thanks Laura Heraty for copy editing.

\section{REFERENCES}

1. Emery P, Symmons DP. What is early rheumatoid arthritis?: definition and diagnosis. Baillieres Clin Rheumatol 1997;11:13-26.

2. Aletaha D, Huizinga TW. The use of data from early arthritis clinics for clinical research. Best Pract Res Clin Rheumatol 2009;23:117-23.

3. Saraux A, Berthelot JM, Chales G, Le Henaff C, Thorel JB, Hoang S, et al. Ability of the American College of Rheumatology 1987 criteria to predict rheumatoid arthritis in patients with early arthritis and classification of these patients two years later. Arthritis Rheum 2001;44:2485-91.

4. Banal F, Dougados M, Combescure C, Gossec L. Sensitivity and specificity of the American College of Rheumatology 1987 criteria for the diagnosis of rheumatoid arthritis according to disease duration: a systematic literature review and meta-analysis. Ann Rheum Dis 2009;68:1184-91.

5. Hulsemann JL, Zeidler H. Diagnostic evaluation of classification criteria for rheumatoid arthritis and reactive arthritis in an early synovitis outpatient clinic. Ann Rheum Dis 1999;58:278-80.

6. Saraux A, Guedes C, Allain J, Baron D, Youinou P, Le Goff P. From classification criteria to diagnostic criteria for rheumatoid arthritis. Rev Rhum Engl Ed 1996;63:159-61.

7. Morvan J, Berthelot J, Devauchelle-Pensec V, Jousse-Joulin S, Le Henaff-Bourhis C, Hoang S, et al. Changes over time in the diagnosis of rheumatoid arthritis in a 10 year cohort. J Rheumatol 2009;36:2428-34.

8. Arnett FC, Edworthy SM, Bloch DA, McShane DJ, Fries JF, Cooper NS, et al. The American Rheumatism Association 1987 revised criteria for the classification of rheumatoid arthritis. Arthritis Rheum 1988;31:315-24.

9. Vitali C, Bombardieri S, Jonsson R, Moutsopoulos HM, Alexander EL, Carsons SE, et al. Classification criteria for Sjogren's syndrome: a revised version of the European criteria proposed by 
the American-European Consensus Group. Ann Rheum Dis 2002;61:554-8.

10. Yamaguchi M, Ohta A, Tsunematsu T, Kasukawa R, Mizushima Y, Kashiwagi H, et al. Preliminary criteria for classification of adult Still's disease. J Rheumatol 1992;19:424-30.

11. Combe B, Benessiano J, Berenbaum F, Cantagrel A, Daures JP, Dougados M, et al. The ESPOIR cohort: a ten-year follow-up of early arthritis in France: methodology and baseline characteristics of the 813 included patients. Joint Bone Spine 2007;74:440-5.

12. Benhamou M, Rincheval N, Roy C, Foltz V, Rozenberg S, Sibilia J, et al. The gap between practice and guidelines in the choice of first-line disease modifying antirheumatic drug in early rheumatoid arthritis: results from the ESPOIR cohort. J Rheumatol 2009;36:934-42.
13. Visser H. Early diagnosis of rheumatoid arthritis. Best Pract Res Clin Rheumatol 2005;19:55-72.

14. Visser H, le Cessie S, Vos K, Breedveld FC, Hazes JM. How to diagnose rheumatoid arthritis early: a prediction model for persistent (erosive) arthritis. Arthritis Rheum 2002;46:357-65.

15. van Dongen H, van Aken J, Lard LR, Visser K, Ronday HK, Hulsmans HM, et al. Efficacy of methotrexate treatment in patients with probable rheumatoid arthritis: a double-blind, randomized, placebo-controlled trial. Arthritis Rheum 2007;56:1424-32.

J Rheumatol 2009;36:2375-7; doi:10.3899/jrheum.091045 